\title{
QUALIDADE DE VIDA EM PESSOAS COM ÚLCERA VENOSA E AS CARACTERÍSTICAS E SINTOMAS ASSOCIADOS À FERIDA
}

\author{
Uiara Aline de Oliveira Kaizer ${ }^{1, *}$ (D), Elaine Aparecida Rocha Domingues² (D), \\ Ana Beatriz de Toledo Saib Paganelli² (D)
}

\begin{abstract}
RESUMO
Objetivo: avaliar a qualidade de vida de pessoas com úlcera venosa e correlacionar com as características clínicas e sintomas associados à ferida. Método: estudo quantitativo, transversal e analítico. Para as análises das associações das variáveis qualitativas foram aplicados os testes Qui-quadrado e exato de Fisher, e para as variáveis quantitativas, o coeficiente de correlação de Spearman. Utilizou-se o instrumento Pressure Ulcer Scale for Healing para a avaliação da ferida e o Freiburg Life Quality Assessment Wound-wk para análise da qualidade de vida. Fizeram parte do estudo 103 sujeitos com tempo de feridas superior a seis semanas, de etiologia venosa. Resultados: houve correlação das características clínicas da úlcera venosa e sintomas associados com a qualidade de vida. Nas dimensões sintomas físicos, pessoas com úlcera venosa apresentraram pior escore em relação ao sintoma dor $(p<0,0001)$. Associadas a isso, as características clínicas como a aparência $(p<0,0001)$ e a área $(p=0,0037)$ da ferida também manifestaram correlação. Em relação à escala de avaliação da ferida (área, tecido na ferida e exsudato), o pior escore interferiu nos sintomas físicos. Conclusão: a dor, tamanho da ferida e seu aspecto influenciam negativamente na qualidade de vida de pessoas com úlcera venosa.
\end{abstract}

DESCRITORES: Úlcera varicosa. Qualidade de vida. Cicatrização. Enfermagem. Estomaterapia.

\section{QUALITY OF LIFE IN PEOPLE WITH VENOUS ULCERS AND THE CHARACTERISTICS AND SYMPTOMS ASSOCIATED WITH THE WOUND}

\begin{abstract}
Objective: to evaluate the quality of life of people with venous ulcers and correlate with the clinical characteristics and symptoms associated with the wound. Method: quantitative, cross-sectional and analytical study. For analyzes of associations of qualitative variables, the Chi-square and Fisher's exact tests were applied, and for quantitative variables, Spearman's correlation coefficient. The Pressure Ulcer Scale for Healing instrument was used to assess the wound and the Freiburg Life Quality Assessment Wound-wk for quality of life analysis. The study included 103 subjects with a wound time of more than six weeks, of venous etiology. Results: there was a correlation between the clinical characteristics of the venous ulcer and symptoms associated with quality of life. In the physical symptoms dimensions, people with venous ulcers had a worse score in relation to the pain symptom $(p<0.0001)$. Associated with this, clinical characteristics such as the appearance $(p<0.0001)$ and the area
\end{abstract}

1. Universidade Estadual de Campinas - Faculdade de Enfermagem - Departamento de Enfermagem - Sorocaba (SP), Brasil. 2. Universidade Vale do Rio Verde - Faculdade de Enfermagem - Departamento de Enfermagem - Três Corações (MG), Brasil. *Autora correspondente: uiara_oliveira@hotmail.com

Editor de Seção: Juliano Teixeira Moraes

Recebido: Nov. 04, 2020 | Aceito: Dez. 14, 2020

Como citar: Kaizer UOA; Domingues EAR; Paganelli ABTS. Qualidade de vida em pessoas com úlcera venosa e as características e sintomas associados à ferida. ESTIMA, Braz. J. Enterostomal Ther., 2020, 19: e0121. https://doi.org/10.30886/estima.v19.968_PT 
$(p=0.0037)$ of the wound also showed a correlation. Regarding the wound assessment scale (area, tissue in the wound and exudate), the worst score interfered with physical symptoms. Conclusion: the pain, size of the wound and its aspect negatively influences the quality of life of people with venous ulcers.

DESCRIPTORS: Varicose ulcer. Quality of life. Healing. Nursing. Stomatherapy.

\title{
CALIDAD DE VIDA EN PERSONAS CON ÚLCERAS VENOSAS Y CARACTERÍSTICAS Y SÍNTOMAS ASOCIADOS A LA HERIDA
}

\begin{abstract}
RESUMEN
Objetivo: evaluar la calidad de vida de las personas con úlceras venosas y correlacionarlas con las características clínicas y síntomas asociados a la herida. Método: estudio cuantitativo, transversal y analítico. Para los análisis de asociaciones de variables cualitativas se aplicaron las pruebas de Chi-cuadrado y exacta de Fisher y para las cuantitativas, el coeficiente de correlación de Spearman. Se utilizó el instrumento Escala de cicatrización de úlceras por presión (PUSH) para la evaluación de heridas y la Evaluación de la calidad de vida de Freiburg Wound -wk para el análisis de la calidad de vida. El estudio incluyó a 103 sujetos con un tiempo de herida mayor de seis semanas, de etiología venosa. Resultados: hubo correlación entre las características clínicas de la úlcera venosa y los síntomas asociados a la calidad de vida. En la dimensión de síntomas físicos, las personas con UV presentaron peor puntuación ante el síntoma de dolor $(p<0,0001)$. En asociación, características clínicas como el aspecto $(p<0,0001)$ y el área $(p=$ 0,0037 ) de la herida también mostraron una correlación. En cuanto a la escala de valoración de la herida (área, tejido de la herida y exudado), la peor puntuación interfirió con los síntomas físicos. Conclusión: el dolor, el tamaño de la herida y su aspecto influyen negativamente en la calidad de vida de las personas con úlceras venosas.
\end{abstract}

DESCRIPTORES: Úlcera venosa. Calidad de vida. Curación. Enfermería. Estomaterapia.

\section{INTRODUÇÃO}

As feridas crônicas representam um grave problema de saúde pública, pois apresentam elevados índices de incidência e prevalência no cenário mundial. Esse contexto duplica em taxas quando se refere aos indivíduos com idade igual ou superior a 65 anos, primordialmente do gênero feminino acima de 70 anos $^{1}$.

As úlceras de perna prevalentes são as úlceras venosas (UV) e com altos índices de recidivas, ocasionadas pela hipertensão venosa e caracterizada pelo processo de reparação tecidual lentificado. Acometem primordialmente a região perimaleolar, e apresentam como características clínicas o edema, eczema, veias varicosas, bordas irregulares e exsudato em grande quantidade ${ }^{2}$.

O processo fisiopatológico da UV está relacionado a uma combinação de mecanismos microscópios e macroscópicos. As modificações macroscópicas concernem-se ao desenvolvimento de veias varicosas, alteração nas estruturas da veia e anomalias celulares que danificam a função do sistema venoso. Tal situação decorre devido a fatores genéticos e hipertensão venosa $^{3}$. A hipertensão venosa ocasiona uma resposta inflamatória crônica devido ao extravasamento de macromoléculas, como hemácias, fibrinogênio e ferro, que sucedem em degradação, exacerbando a resposta inflamatória local. Com o processo inflamatório crônico, a quimiotaxia atrai os leucócitos até o local e liberam abundantes citocinas pró-inflamatórias que elevam o dano tissular da pele ${ }^{3}$.

O impacto que a UV suscita é notável nas pessoas de qualquer faixa etária, principalmente devido à sua cronicidade e altas taxas de recidivas. Sua presença reflete de maneira negativa na qualidade de vida (QV) devido ao impacto biopsicoespiritual e econômico para os indivíduos e seus familiares ${ }^{4}$.

As definições que permeiam QV são muitas, em diferentes áreas e estão relacionadas à sua capacidade de definir e mensurar grandes dimensões, sendo direcionadas ao campo da saúde, recebendo a denominação de Qualidade de Vida 
Relacionada à Saúde (QVRS), incorporando vários aspectos, como o estado de saúde, sintomas físicos, incapacidades, fatores emocionais e bem-estar 5 .

No aspecto físico e no psicossocial, as pessoas com UV são afetadas pela dor e dificuldades de deambulação, tornam-se limitadas às atividades domésticas e laborais. Ademais, há comprometimento do estado emocional e social, pois alguns indivíduos sentem vergonha da situação. Desse modo, eles necessitam de cuidados voltados aos problemas, com o objetivo de reestabelecer a QV para retornar às suas atividades diárias ${ }^{4,6,7}$.

Estudos evidenciam que a UV interfere na QV, principalmente, nos aspectos físicos alusivos às características clínicas da UV (área da ferida, exsudato e aparência da ferida) e os sintomas associados, como a principal queixa, a dor ${ }^{8,9}$. Dessa maneira, faz-se necessário que os profissionais prestem assistência integral ao paciente com UV, identificando quais as peculiaridades da UV e os sintomas associados que impactam negativamente na QV para nortear a sua assistência.

Mediante o contexto, emergiu a seguinte questão norteadora: as características clínicas da ferida e os sintomas associados influenciam na QV de pessoas com UV? Portanto, o objetivo do estudo é avaliar a QV de pessoas com UV e correlacionar com as características clínicas e sintomas associados à ferida.

\section{MÉTODO}

Trata-se de estudo observacional transversal de série de casos, com dados secundários de uma pesquisa primária relacionada à avaliação da $\mathrm{QV}$ em pessoas com UV submetidas a uma intervenção ${ }^{10}$. O estudo foi realizado em três unidades de atendimento especializado em pessoas com feridas, no período de novembro de 2016 a julho de 2017.

As unidades de atendimento estão localizadas no Sul de Minas Gerais e São Paulo. O protocolo no atendimento das pessoas com UV consiste na avaliação da UV pelo enfermeiro e médico vascular. Os pacientes realizam exames de imagem e Índice Tornozelo-Braquial (ITB) para o diagnóstico da UV, e posteriormente é realizada prescrição conforme características da ferida, associada à terapia compressiva.

Os participantes do estudo foram recrutados nas unidades referidas, por meio de uma lista solicitada pela pesquisadora à enfermeira responsável pela unidade, contendo o nome do paciente, o número de prontuário e o seu telefone. O recrutamento foi realizado conforme agendamento do paciente na unidade de origem para acompanhamento rotineiro. Os pacientes foram selecionados de acordo com a chegada à unidade para tratamento, antes da realização do curativo, na sala de espera.

Participaram do estudo pessoas com UV, seguindo os seguintes critérios: pacientes de ambos os sexos e com ITB entre 0,8 e 1,3 mmHg, com tempo de UV superior a 6 semanas. Pacientes com diagnóstico de diabetes mellitus e úlceras infectadas foram excluídos do estudo.

Para o cálculo amostral, levou-se em consideração a metodologia de um modelo de Anova de medidas repetidas, assumido um nível de significância de 5\%, um poder de teste de $80 \%$ e um tamanho de efeito de 0,25 , o qual pode ser considerado um tamanho de efeito de grau médio. O cálculo resultou em uma amostra de 82 indivíduos. Contudo, considerando uma taxa de $20 \%$ para possíveis perdas, o tamanho amostral totalizou 98 sujeitos. Utilizou-se para a realização do cálculo amostral o software $G^{*}$ Power 3.1.9.2.

Os dados foram coletados por meio de entrevista em papel impresso e de maneira individual pela pesquisadora principal, em local privativo. Utilizou-se o instrumento de dados sociodemográficos e de saúde para caracterizar os participantes, desenvolvido pela pesquisadora.

O instrumento Pressure Ulcer Scale for Healing (PUSH) foi utilizado para a avaliação das feridas ${ }^{11}$. O PUSH está dividido em três subescalas: área da ferida (escore varia de 0 a 10); quantidade de exsudato, classificado como ausente, pequeno, moderado e grande quantidade (escore varia de 0 a 3); e aparência do leito (escore varia de 0 a 4). O escore total é obtido por meio da somatória das subescalas, permeia de 0 (ferida cicatrizada) a 17 (pior estado).

Para análise da dor, foi utilizada a escala numérica da dor. Trata-se de uma escala de 11 pontos, que é marcada de 0 a 10 , em que os pontos finais são os extremos, sendo 0 (nenhuma dor) e 10 (pior dor imaginável) ${ }^{12}$. 
Para avaliar a QV foi utilizado o instrumento FLQA-wk- versão abreviada, adaptado e validado no Brasil $(2013)^{13}$. O instrumento é específico para avaliar a QVRS de pessoas com úlceras crônicas de distintas etiologias. A escala contempla seis domínios: sintomas físicos (dor, insônia, prurido e odor da ferida), vida diária, vida social, bem-estar psicológico, tratamento da ferida e satisfação (saúde, ferida e tratamento). Ao final do instrumento existe a avaliação da aparência da ferida na visão do paciente, a qual ele pontua de zero (pior estado da ferida) a dez (bom estado da ferida).

O escore total é realizado pela média de cada resposta após a recodificação da escala satisfação, ou seja, os valores necessitam ser invertidos para o cálculo. Já o escore total é computado por meio dos valores médios de cada domínio. O escore de QV varia de 1 a 1,25 (ótima QV); 1,26 a 2,5 (média QV); 2,6 a 3,75 (regular QV); e 3,76 a 5 (pior QV).

Para mensurar a área da lesão foi realizado o registro fotográfico da UV com câmera digital de 8 megapixels, abertura f/2.4, flash Diodo Light Emitting (LED) e utilizada a planimetria computadorizada para cálculo da área (Texas Health Science Center em San Antonio Image Tool, versão 3.0, baixado de www.ddsdx.uthsca.edu/dig/itdesc.html).

A coleta de dados foi realizada após a assinatura do Termo de Consentimento Livre e Esclarecido (TCLE) e aprovação do Comitê de Ética sob parecer nº 1.860.409. Respeitaram-se os preceitos éticos da resolução 466/12.

Os dados foram inseridos no Microsoft Office Excel 2010 e, em seguida, avaliados usando versão 9.4 do software estatístico Statistical Analysis System (SAS). Para a análise, utilizou-se para as variáveis contínuas, a média e o desvio padrão, e para as variáveis categóricas, frequências relativa e absoluta. Nas associações entre as variáveis qualitativas foram realizados os testes Qui-quadrado e exato de Fisher (em que os pressupostos não foram atendidos). Para as correlações entre as variáveis quantitativas foi aplicado o coeficiente de correlação de Spearman. Considerou-se um nível de significância quando o p-valor for menor do que 0,0250 .

\section{RESULTADOS}

Participaram do estudo 103 pessoas com UV, contudo, 33 foram excluídas conforme Fig. 1. Como apresenta a Tabela 1, a idade média foi de 66,5 anos, com predomínio do sexo masculino $(58,5 \%)$, sendo que $54,2 \%$ viviam sem companheiros, a renda permeava 1,5 salários mínimos e os indivíduos apresentavam escolaridade de 3,8 anos de estudos.

Apresentou em média 57,2 meses de ocorrência de ferida, ITB com média de 1,0, uso de compressão elástica (88,5\%) e o tratamento prevalente foi uso de polihexametileno de biguanida, alginato de cálcio e hidrogel. A média de intensidade da dor foi de 5,2 pontos e a área da ferida foi de $11,9 \mathrm{~cm}^{2}$.

Em relação à escala PUSH, o escore total foi de 10,9; a área da ferida apresentou escore de 6,6; a quantidade de exsudato e o tecido predominante no leito da úlcera apresentaram escores, respectivamente, de 1,9 e 2,3.

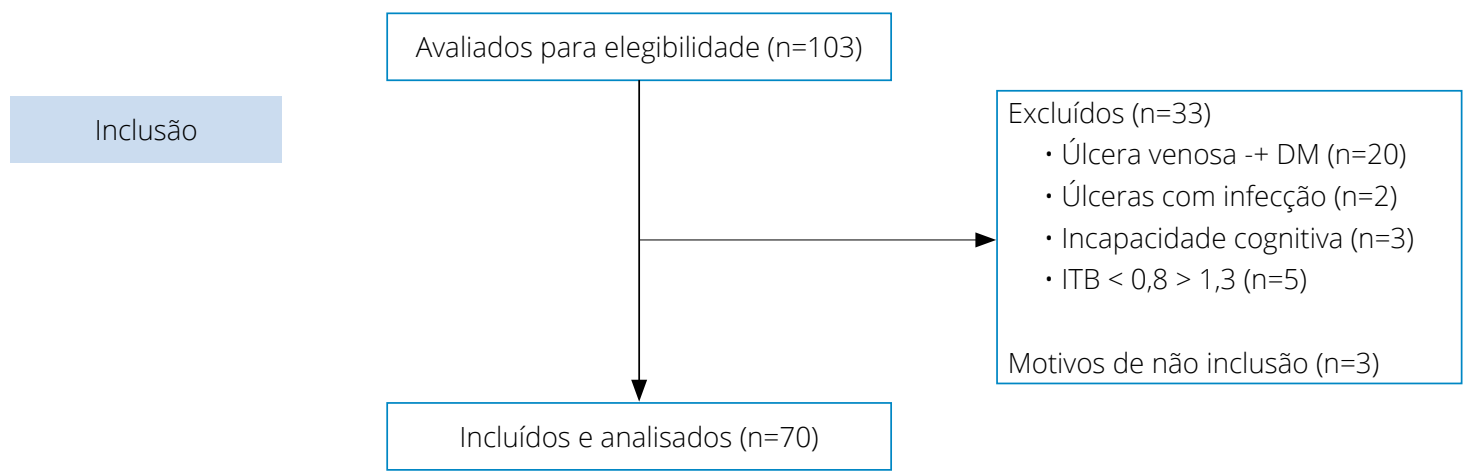

DM = Diabetes Mellitus; ITB = Índice Tornozelo-Braquial

Figura 1. Fluxograma dos participantes - Três Corações (MG), Brasil - 2019. 
Tabela 1. Dados pessoais e clínicos de pessoas com úlceras venosas (n=70). Três Corações (MG), Brasil - 2019.

\begin{tabular}{|c|c|c|c|c|c|}
\hline Variável & $\begin{array}{c}\text { Frequência } \\
\text { absoluta }\end{array}$ & $\begin{array}{c}\text { Frequência } \\
\text { relativa }\end{array}$ & $\begin{array}{l}\text { Média } \\
\text { (DP) }\end{array}$ & $\begin{array}{l}\text { Valor } \\
\text { mínimo }\end{array}$ & $\begin{array}{l}\text { Valor } \\
\text { máximo }\end{array}$ \\
\hline Idade (anos) & & & $66,5(12,8)$ & 38 & 93 \\
\hline \multicolumn{6}{|l|}{ Sexo } \\
\hline Feminino & 29 & $41,4 \%$ & & & \\
\hline Masculino & 41 & $58,5 \%$ & & & \\
\hline \multicolumn{6}{|l|}{ Estado civil } \\
\hline Com companheiro & 32 & $45,7 \%$ & & & \\
\hline Sem companheiro & 38 & $54,2 \%$ & & & \\
\hline \multicolumn{6}{|l|}{ Tratamento } \\
\hline Petrolatum, placa de hidrocoloide e papaína & 12 & $17,1 \%$ & & & \\
\hline Alginato de cálcio, hidrogel e phmb & 56 & $80,0 \%$ & & & \\
\hline Outros & 2 & $2,8 \%$ & & & \\
\hline \multicolumn{6}{|l|}{ Compressão } \\
\hline Elástica & 62 & $88,5 \%$ & & & \\
\hline Inelástica & 8 & $11,4 \%$ & & & \\
\hline Renda salarial & & & $1,5(0,01)$ & 1 & 5 \\
\hline Escolaridade (anos) & & & $3,8(0,1)$ & 0 & 13 \\
\hline ITB (mmHg) & & & $1,0(0,007)$ & 0,9 & 1,2 \\
\hline Tempo de feridas (meses) & & & $57,2(1,8)$ & 2 & 300 \\
\hline Dor $(0-10)$ & & & $5,2(3,7)$ & 0 & 10 \\
\hline Área da ferida $\left(\mathrm{cm}^{2}\right)$ & & & $11,9(16,7)$ & 0,17 & 92,41 \\
\hline Área - PUSH & & & $6,6(2,6)$ & 1 & 10 \\
\hline Exsudato - PUSH & & & $1,9(0,6)$ & 1 & 3 \\
\hline Tecido - PUSH & & & $2,3(0,5)$ & 2 & 4 \\
\hline Escore total PUSH & & & $10,9(3,1)$ & 5 & 16 \\
\hline
\end{tabular}

DP = Desvio padrão; PHMB = Polihexametileno biguanida; ITB = Índice Tornozelo-Braquial; PUSH = Pressure U/cer Scale for Healing.

A Tabela 2 destaca o escore de QV e seus domínios. O escore total foi de 2,4 e os domínios tratamento $(2,7)$ e vida diária $(2,7)$ foram os que mais interferiram negativamente na QV, classificada como regular QV. Na escala estado da ferida, a média demarcada foi de 6,8 .

A Tabela 3 demonstra a correlação das características clínicas da UV e os sintomas associados à QV. Nas dimensão sintomas físicos, pessoas com UV apresentaram pior escore em relação ao sintoma dor ( $\mathrm{p}<0,0001)$. Associadas a isso, as características clínicas, como a aparência $(\mathrm{p}<0,0001)$ e a área $(\mathrm{p}=0,0037)$ da ferida também manifestaram correlação.

No item satisfação, a melhora da dor $(\mathrm{p}=0,0162)$ e os aspectos da ferida $(\mathrm{p}<0,0001)$ apresentaram correlação significativa. De modo que quanto maior o escore da aparência da ferida, menor o escore do domínio satisfação e vice-versa. Essa relação inversamente proporcional é determinada pelo coeficiente de correlação de Spearman negativo, mostrando que quando um escore aumenta ou outro diminui. 
Na dimensão vida diária, o sintoma dor $(\mathrm{p}=0,0024)$ demonstrou impacto no dia a dia dos indivíduos. Por outro lado, nos domínios vida social, bem-estar psicológico e tratamento não houve características da ferida que interferissem diretamente nesses aspectos.

Referente ao escore total de QV, o sintoma dor ( $p<0,0001)$, aparência da ferida ( $p=0,0004)$ e escore do PUSH ( $p=0,0220)$ interferem negativamente na QV de pessoas com UV.

Tabela 2. Escore de qualidade de vida de pacientes com úlceras venosas (n=70). Três Corações (UF), Brasil - 2019.

\begin{tabular}{lc}
\hline Domínios & Média (DP) \\
\hline Sintomas físicos & $2,61(0,55)$ \\
\hline Vida diária & $2,72(0,16)$ \\
\hline Vida social & $2,17(0,48)$ \\
\hline Bem-estar psicológico & $1,94(0,30)$ \\
\hline Tratamento & $2,74(0,58)$ \\
\hline Satisfação & $2,32(0,37)$ \\
\hline Escore total & $2,45(0,75)$ \\
\hline Ferida (0-10) & $6,8(2,5)$ \\
\hline
\end{tabular}

DP = Desvio padrão

Tabela 3. Correlação das características clínicas da úlcera venosa e sintomas associados à qualidade de vida. Três Corações (MG), Brasil - 2019.

\begin{tabular}{|c|c|c|c|c|}
\hline Qualidade de vida & $\begin{array}{l}\text { Dor } \\
(0-10)\end{array}$ & $\begin{array}{l}\text { Ferida } \\
(0-10)\end{array}$ & $\begin{array}{l}\text { Área da ferida } \\
\left(\mathrm{cm}^{2}\right)\end{array}$ & $\begin{array}{l}\text { Escore - Total } \\
\text { (PUSH) - (0-17) }\end{array}$ \\
\hline \multirow{2}{*}{ Escore - sintomas físicos } & 0,7685 & $-0,5251$ & 0,3428 & 0,3519 \\
\hline & $p<0,0001$ & $p<0,0001$ & $p=0,0037$ & $p=0,0030$ \\
\hline \multirow{2}{*}{ Escore - vida diária } & 0,3886 & $-0,2741$ & 0,2125 & 0,2321 \\
\hline & $p=0,0024$ & $p=0,0260$ & $p=0,0775$ & $p=0,0549$ \\
\hline \multirow{2}{*}{ Escore - vida social } & 0,2482 & $-0,2002$ & 0,0660 & 0,0857 \\
\hline & $p=0,0581$ & $p=0,1071$ & $p=0,5874$ & $p=0,4841$ \\
\hline \multirow{2}{*}{ Escore - bem-estar psicológico } & 0,2114 & $-0,2248$ & 0,0916 & 0,1213 \\
\hline & $p=0,1079$ & $p=0,0696$ & $p=0,4507$ & $p=0,3209$ \\
\hline \multirow{2}{*}{ Escore - tratamento } & 0,1384 & $-0,2083$ & 0,0504 & 0,0969 \\
\hline & $p=0,2960$ & $p=0,0933$ & $p=0,6787$ & $p=0,4284$ \\
\hline \multirow{2}{*}{ Escore - satisfação } & 0,3118 & $-0,5773$ & 0,2502 & 0,3221 \\
\hline & $p=0,0162$ & $p<0,0001$ & $p=0,0367$ & $p=0,0070$ \\
\hline \multirow{2}{*}{ Escore - total (FLQA) } & 0,5057 & $-0,4238$ & 0,2442 & 0,2755 \\
\hline & $p<0,0001$ & $p=0,0004$ & $p=0,0416$ & $p=0,0220$ \\
\hline
\end{tabular}

Coeficiente de correlação de Spearman e p-valor $<0,025$

PUSH = Pressure UIcer Scale for Healing; FLQA = Freiburg Life Quality Assessment.

A Tabela 4 demonstra a relação dos domínios do PUSH e da QV, evidenciando que não houve correlação significativa entre eles. 
Tabela 4. Correlação das características clínicas da lesão (Pressure Ulcer Scale for Healing) na qualidade de vida. Três Corações (MG), Brasil - 2019.

\begin{tabular}{|c|c|}
\hline Variável & QV \\
\hline \multirow{2}{*}{ Área Total } & $-0,0615$ \\
\hline & $p=0,6268$ \\
\hline \multirow{2}{*}{ Exsudato } & 0,0685 \\
\hline & $p=0,5878$ \\
\hline \multirow{2}{*}{ Tecido no leito } & $-0,1213$ \\
\hline & $p=0,3356$ \\
\hline
\end{tabular}

Coeficiente de correlação de Spearman e p-valor $<0,025$

QV= Qualidade de vida.

\section{DISCUSSÃO}

O estudo evidenciou maior frequência de UV em idosos do sexo masculino e com baixa renda e nível de escolaridade. Tal cenário corrobora estudos internacionais e nacionais sobre o perfil dessa população, e enfatiza que pessoas com UV necessitam de atenção especializada no que concerne à assistência integral, propendendo ao reparo tecidual e redução das recorrências e, consequentemente, na melhoria da $\mathrm{QV}^{8,9,13-15}$.

Nessa pesquisa, as pessoas com UV apresentaram baixa QV, influenciadas pelo impacto que a ferida ocasiona na vida diária, seus sintomas físicos, principalmente, a dor e pelos sinais clínicos peculiares presentes (tamanho e a aparência da ferida $)^{13}$. Estudos que relacionam a dor e a $\mathrm{QV}$ destacam que a atenção especializada à dor é um cuidado preditor para a melhoria da $\mathrm{QV}$, no que incumbe a sua avaliação, a utilização de escalas validadas e a identificação de fatores que aliviam ou pioram o sintoma são os cuidados de enfermagem fundamentais para o controle do sintoma ${ }^{16-18}$.

Outro estudo ${ }^{19}$, que também analisou a dor em casos de UV, encontrou que devido à queixa de dor intensa pelas pessoas com UV, além do uso de medicação para controlar esse sintoma, outros métodos não farmacológicos são necessários, dentre eles, uma boa relação familiar, social e de lazer. Caso contrário, as pessoas podem se isolar da sociedade, desenvolver baixa autoestima e até depressão, comprometendo ainda mais a sua QV.

Atrelado ao sintoma físico, indivíduos com UV apresentam dificuldade para realizar as tarefas do seu dia a dia, pois as feridas acometem especificamente os membros inferiores e logo suscitam importantes limitações físicas. Atreladas a isso, feridas extensas e com aparência ruim prejudicam ainda mais a vida diária dos pacientes, além da vergonha e isolamento social ${ }^{7}$.

A pesquisa corrobora ao destacar que a profundidade, tamanho e a presença de exsudato nas UV ocasionam incapacidade e limitações. Os pacientes perdem a QV, pois evitam o contato social, deixam de realizar suas atividades, ficam isolados, e tal situação leva a quadros depressivos. Ademais, o fato de existir um exsudato que pode apresentar um odor fétido por conta de infecções, ou mesmo a aparência da lesão pode resultar em repulsas, como não se alimentar adequadamente, o que gera comprometimento no processo cicatricial e uma baixa $\mathrm{QV}^{20}$.

Além da dor, a aparência da ferida e a sua extensão foram aspectos clínicos que impactaram de maneira negativa a QV dos participantes com UV. Portanto, vale destacar a importância do profissional de enfermagem nesse contexto, uma vez que detém a fundamentação teórica e a habilidade para prestar assistência aos pacientes com alteração da integridade da pele. Para auxiliar na redução do tempo da reparação tecidual, o profissional deve utilizar ferramentas de avaliação para acompanhar a evolução bem como conhecer as distintas tecnologias e inovações disponíveis no mercado para o tratamento de feridas em geral, e analisar os fatores sistêmicos e locais que interferem na cicatrização ${ }^{21}$.

A principal contribuição deste estudo para a pesquisa em UV foi identificar quais são os fatores clínicos que mais afetam a QV das pessoas com UV crônicas e quais os domínios que são mais afetados, para melhor planejamento da assistência prestada e também desenvolver programas voltados para assistência integral desses casos, com informações 
para subsidiar as políticas de saúde, de prevenção e tratamento dessas pessoas, visando melhorar a sua QV. Vale salientar que o questionário utilizado para a pesquisa é um instrumento específico que avalia a QV das pessoas com UV e aborda a dimensão do tratamento da ferida, um dos itens que mais influenciou negativamente na $\mathrm{QV}$ dos participantes, o que difere dos demais instrumentos da literatura. Ademais, os domínios sintomas físicos e atividade diária apresentaram impacto na vida do paciente com UV.Todas essas alterações interferem na vida diária do paciente com ferida, e um planejamento eficaz da assistência promove a reparação tecidual e melhora a percepção, favorecendo o bem-estar e QV da pessoa com UV ${ }^{9}$.

Como limitação deste estudo, destaca-se que os dados são secundários de uma pesquisa primária relacionada ao estilo de vida em pacientes com UV, contudo, o objetivo estava relacionado à QV. Ademais, destaca-se o viés da coleta de dados de QV por meio de entrevista e não por autopreenchimento, pois os participantes podem omitir informações por sentirem-se constrangidos em responder as perguntas.

\section{CONCLUSÃO}

Pessoas com UV apresentam QV diminuída, pois a presença e as características clínicas da ferida (extensão da ferida, quantidade de exsudato e presença de tecido no leito) e o sintoma dor prejudicam a vida diária do paciente e seus familiares. $\mathrm{O}$ tratamento oferecido à pessoa com UV e seus sintomas físicos foram as dimensões que pior caracterizaram a $\mathrm{QV}, \operatorname{logo}$ os profissionais da saúde necessitam explanar de forma clara e objetiva a importância do tratamento e as orientações de autocuidado para que a pessoa compreenda e siga as recomendações para alcance da reparação tecidual. Ao correlacionar os sintomas e as características clínicas da UV, evidenciou-se que os sintomas físicos, principalmente, a intensidade da dor, área e aparência da ferida são aspectos desfavoráveis que interferem negativamente na QV.

\section{CONTRIBUIÇÃO DOS AUTORES}

Conceitualização: Domingues EAR; Metodologia: Domingues EAR; Investigação: Domingues EAR e Kaizer UAO; Redação - Primeira versão: Paganelli ABTS, Kaizer UAO e Domingues EAR; Redação - Revisão \& Edição: Paganelli ABTS, Kaizer UAO e Domingues EAR; Supervisão: Kaizer UAO e Domingues EAR.

\section{DISPONIBILIDADE DE DADOS DE PESQUISA}

Dados serão enviados mediante solicitação.

\section{FINANCIAMENTO}

Não se aplica.

\section{AGRADECIMENTOS}

Não se aplica.

\section{REFERÊNCIAS}

1. Pérez MB, López-Casanova P, Lavín RS, Torre HG, Verdú-Soriano J. Epidemiology of venous leg ulcers in primary health care: Incidence and prevalence in a health centre-A time series study (2010-2014). Int Wound J 2019;16(1):256-65. https://doi. org/10.1111/iwj.13026

2. Sánchez-Nicolat NE y cols. Úlceras venosas. Rev Mex Angiol 2019;47(1):26-38. 
3. Crawford JM, Lal BK, Durán WN, Pappas PJ. Pathophysiology of venous ulceration. J Vasc Surg Venous Lymphat Disord 2017 jul;5(4):596-605. https://doi.org/10.1016/j.jvsv.2017.03.015

4. Araújo RO, Silva DC, Souto RQ, Pergola-Marconato AM, Costa IKF, Torres GV. Impacto de úlceras venosas na qualidade de vida de indivíduos atendidos na atenção primária. Aquichan 2016:16(1):56-66. https://doi.org/10.5294/aqui.2016.16.1.7

5. Karimi M, Brazier J. Health, Health-Related Quality of Life, and Quality of Life: What is the Difference? Pharmacoeconomics 2016;34(7):645-9. https://doi.org/10.1007/s40273-016-0389-9

6. Barbosa MLG, Salomé GM, Ferreira LM. Avaliação da ansiedade e da depressão em paciente com úlcera venosa tratados com acupuntura. Rev Enferm UFP on line 2017; [Citado 2018 set 6]; 11(Supl.9):3574-82. Disponível em: https://periodicos.ufpe.br/ revistas/revistaenfermagem/article/download/234488/27686

7. Joaquim FL, Silva RMCRA, Garcia-Caro MP, Cruz-Quintana F, Pereira ER. Impact of venous ulcers on patients' quality of life: an integrative review. Rev Bras Enferm 2018 Jul-Aug;71(4):2021-9. https://doi.org/10.1590/0034-7167-2017-0516

8. Santos KFR, Silva PR, Ferreira VT, Domingues EAR, Simões IRA, Lima RS et al. Quality of life of people with chronic ulcers. J Vasc Nurs 2016 Dec;34(4):131-6. https://doi.org/10.1016/j.jvn.2016.06.003

9. Torres SMSSO, Araújo RO, Costa IKF, Tibúrcio MP, Sousa AJG, Pergola-Marconato AM et al. Health-related quality of life in patients with venous leg ulcer treated in primary care in Brazil and Portugal. PLoS One 2018;13(4):e0195990. https://doi. org/10.1371/journal.pone.0195990

10. Domingues EAR, Kaizer UAO, Lima MHM. Effectiveness of the strategies of an orientation programme for the lifestyle and wound-healing process in patients with venous ulcer: A randomised controlled trial. Int Wound J 2018;15(5):798-806. https:// doi.org/10.1111/iwj.12930

11. Santos VLCG, Carvalho VF. Reapresentando o instrumento Pressure Ulcer Scale for Healing (PUSH) para avaliação de úlceras por pressão e úlceras crônicas de perna. ESTIMA, Braz J Enterestomal Ther 2009;7(2):19-27. Disponível em: https://www. revistaestima.com.br/estima/article/view/256

12. Karcioglu O, Topacoglu H, Dikme O, Dikme O. A systematic review of the pain scales in adults: Which to use? Am J Emerg Med 2018 Apr;36(4):707-14. https://doi.org/10.1016/j.ajem.2018.01.008

13. Domingues EAR, Alexandre NMC, Silva JV. Adaptação cultural e validação do Freiburg Life Quality Assessment-Wound para a língua portuguesa do Brasil. Rev Latino-Am Enfermagem 2016;24:e2684. http://doi.org/10.1590/1518-8345.0289.2684

14. Torre HG, Quintana-Lorenzo ML, Perdomo-Pérez E, Verdú J. Correlation between health-related quality of life and venous leg ulcer's severity and characteristics: a cross-sectional study. Int Wound J 2017;14(2):360-8. https://doi.org/10.1111/iwj.12610

15. Souza EM, Yoshida WB, Melo VA, Aragão JA, Oliveira LAB. Ulcer due to chronic venous disease: a sociodemographic study in northeastern Brazil. Ann Vasc Surg. 2013 Jul;27(5):571-6. https://doi.org/10.1016/j.avsg.2012.07.021

16. Silva DC, Budó MLD, Schimith MD, Salvetti MG, Torres GV. Estratégias pessoais de alívio da dor utilizadas por pacientes com úlcera venosa. Rev Dor 2015;16(2):86-9. https://doi.org/10.5935/1806-0013.20150017

17. Santos VLCG, Oliveira AS, Amaral AFS, Nishi ET, Junqueira JB, Kim SHP. Quality of life in patients with chronic wounds: magnitude of changes and predictive factors. Rev esc enferm USP 2017;51:e03250. https://doi.org/10.1590/s1980-220x2016049603250

18. Lentsck MH, Baratieri T, Trincaus MR, Mattei AP, Miyahara CTS. Quality of life related to clinical aspects in people with chronic wound. Rev Esc Enferm USP 2018;52:e03384. https://doi.org/10.1590/S1980-220X2017004003384

19. Brito D, Xavier V, Santos J, Agra G. Dor em úlcera crônica: perfil sociodemográfico, clínico e terapêutico de pacientes de CuitéPB. J Aging Innovation 2017; [Citado 2018 Set 6]; 6(2):17-31 Disponível em: http://www.journalofagingandinnovation.org/wpcontent/uploads/3-artigo-dor-em-\%C3\%9Alcera-cr\%C3\%94nica-24_04_17.pdf

20. Almeida WA, Ferreira AM, Ivo ML, Rigotti MA, Barcelos LS, Silva ALNV. Fatores associados à qualidade de vida de pessoas com feridas complexas crônicas. Rev Fund Care Online 2018;10(1):9-16. https://doi.org/10.9789/2175-5361.2018.v10i1.9-16

21. Lumbers M. TIMERS: undertaking wound assessment in the community. Br J Community Nurs 2019;24(Sup12):S22-5. https:// doi.org/10.12968/bjen.2019.24.Sup12.S22 